\title{
Étude des phénomènes d'interaction digestive en Rusitec pour des mélanges simples d'aliments concentrés
}

\author{
S Giger-Reverdin, D Sauvant, M Rigault, \\ $P$ Schmidely, MJ François \\ INRA, station de nutrition et alimentation, \\ Institut national agronomique Paris-Grignon, \\ 16. rue Claude-Bernard, 75231 Paris Cedex 05, France
}

\begin{abstract}
Summary - Digestive interaction study in a Rusitec device with binary mixtures of concentrate feeds. Estimation of nutritive value of compound feeds is based on the additivity of their ingredient values. This hypothesis was tested with binary blends in a Rusitec device. Results showed that when energy and protein were available at about the same time, positive interaction appeared after $24 \mathrm{~h}$ on dry matter degradation and on gas production during the first hours of incubation.
\end{abstract}

L'estimation de la valeur nutritive des aliments composés pour les ruminants suppose l'additivité des valeurs des ingrédients des mélanges. La présente étude a eu pour objectif de tester cette hypothèse dans le cas de la dégradation en Rusitec, et donc in vitro et en présence de microorganismes du rumen, de mélanges binaires d'aliments concentiés.

Matériel et méthodes - Les 4 mélanges étudiés ont été fabriqués à partir de matières premières choisies pour leur richesse en glucides (orge-O ou maïs-M), ou en protéines (lupin-L ou gluten meal-G) et pour leur vitesse rapide $(O, L)$ ou lente $(M, G)$ de dégradation dans le rumen. Les 4 combinaisons 2 à 2 de ces matières premières, au sein de mélanges iso-PDI pour une même source azotée, permettent de synchroniser ou de dissocier dans le temps les rythmes de disponibilité des fractions glucidiques ou azotées. Les mélanges OL et ML contiennent $50 \%$ de lupin associé à $40 \%$ d'orge ou de maïs et les mélanges $O G$ et $M G, 30 \%$ de gluten meal et $60 \%$ d'orge ou de maïs. Tous les aliments renferment $10 \%$ de CMV. Ces 4 mélanges ont été étudiés en appareil Rusitec selon une méthodologie comparable à celle utilisée par Durand et al (1988). Cependant, elle en diffère par la grille de broyage des aliments ( 1 vs $0,8 \mathrm{~mm}$ ), le diamètre des pores du tissu (50 vs $150 \mu \mathrm{m}$ ) et le système de mesure des volumes de gaz (Giger-Reverdin, 1990).

Après une phase d'adaptation de $7 \mathrm{j}$, la dégradation de la matière sèche des aliments placés pendant 24 (MS 24) ou 48 (MS 48) h dans les fermenteurs, a été mesurée pendant $4 \mathrm{j}$ consécutifs. Pendant ces jours, les quantités de gaz produit ont été mesurées $1,2,4,6,10$ et $23 \mathrm{~h}$ après le changement des sachets. Les 4 aliments ont été testés simultanément au cours d'une période et 3 périodes ont été effectuées, avec changement d'inoculum et permutation des aliments entre les fermenteurs d'une série à l'autre.

L'hypothèse d'additivité a été testée sur les moyennes de chaque période pour chaque variable en interprétant le dispositif en plan factoriel $2 \times 2$ pour tester les effets de la nature des glucides (OM), des protéines (LG) et l'interaction entre ces 2 effets $(O M \times L G)$.

Résultats et discussion (tableau I) La MS 24 des aliments à base de gluten (OG et MG) est beaucoup plus faible que celle des aliments $\mathrm{OL}$ et $\mathrm{ML}$ à base de lupin (différence moyenne de $18,8 \%$ ). De même, les mélanges à base d'orge sont plus dégradés que 
ceux à base de maîs $(6,6 \%$ de différence). Une interaction statistiquement significative apparaît entre les sources énergétiques et azotées en faveur du mélange OL, c'est-à-dire de celui pour lequel l'énergie et l'azote sont rapidement disponibles. Seule la nature de la source azotée a un effet significatif sur MS 48.

Le production de gaz est fortement influencée par la nature de la source azotée quelle que soit l'heure de prélèvement, alors que la nature glucidique $n$ 'influe que sur les productions de gaz à 6 et $10 \mathrm{~h}$. Une interaction digestive favorable au mélange OL a pu être mise en évidence sur les 4 prélèvements à court terme. Les aliments $\mathrm{OL}$ et $\mathrm{ML}$, qui ont des productions de gaz équivalentes à $23 \mathrm{~h}$ se différencient nettement dans le court terme, avec un démarrage en fermentation plus rapide pour OL. L'aliment $O G$ est celui qui semble démarrer le plus lentement en fermentation à court terme. Ces résultats sont en accord avec les mesures faites in sacco sur ces mêmes échantillons qui montraient que les différences entre échantillons étaient surtout marquées dans les premières heures de l'incubation (Chapoutot et al, 1990). Cependant, la méthode in sacco a discriminé la nature glucidique des aliments plus nettement que l'étude en Rusitec. Ceci peut s'expliquer par une relative carence en azote rapidement disponible des mélanges $O G$ et $M G$ par rapport à $\mathrm{OL}$ et $\mathrm{ML}$, qui se traduit par des teneurs en azote ammoniacal $\left(\mathrm{N}_{\mathrm{NH}_{3}}\right)$ moyennes à court terme, du liquide du fermenteur, faibles pour $O G$ et $M G$ (39 et $31 \mathrm{mg}$ $\mathrm{N}_{\mathrm{NH}_{3}} / 100 \mathrm{ml}$ par rapport à $\mathrm{OL}$ et $\mathrm{ML}$ (203 et 194).

En conclusion, il ressort de cette étude que les différences entre mélanges sont plus marquées au démarrage des fermentations et qu'il y a des interactions notables sur la dégradation de la matière sèche après $24 \mathrm{~h}$ d'incubation, ainsi que sur la production de gaz. D'autres mesures (productions d'acides gras volatils, de protéines microbiennes...) devront être pratiquées pour compléter l'impact des interactions sur la dynamique des fermentations ruminales.

Chapoutot P, Giger S, Sauvant D, Jeantet S (1990) Reprod Nutr Dév (suppl 2) 169S170S, 5th Conf Nutrition and Feeding of Herbivores, 1989

Durand $M$, Dumay C, Beaumatin P, Morel MT (1988) Anim Feed Sci Technol 21, 197204

Giger-Reverdin S (1990) Ann Zootech 39, 173-178

Tableau I. Étude des interactions énergie-azote sur les principaux paramètres fermentaires.

\begin{tabular}{|c|c|c|c|c|c|c|c|}
\hline \multirow[b]{2}{*}{ Aliment } & \multirow[b]{2}{*}{$O L$} & \multirow[b]{2}{*}{$M L$} & \multirow[b]{2}{*}{$O G$} & \multirow[b]{2}{*}{$M G$} & \multicolumn{2}{|c|}{ Effets } & \multirow{2}{*}{$\begin{array}{l}\text { Interaction } \\
O M \times L G\end{array}$} \\
\hline & & & & & $O M$ & $L G$ & \\
\hline 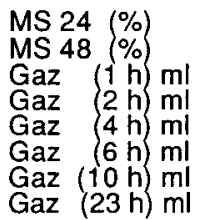 & $\begin{array}{c}68,2 \\
80,4 \\
188 \\
324 \\
684 \\
1029 \\
1519 \\
2340\end{array}$ & $\begin{array}{r}56,0 \\
79,5 \\
157 \\
271 \\
499 \\
764 \\
1418 \\
2237\end{array}$ & $\begin{array}{c}43,9 \\
64,9 \\
77 \\
182 \\
420 \\
703 \\
1276 \\
1667\end{array}$ & $\begin{array}{c}42,8 \\
62,4 \\
165 \\
255 \\
492 \\
708 \\
1073 \\
1662\end{array}$ & $\begin{array}{r}6,6 * \\
1,7 \\
-29 \\
-10 \\
57 \\
130 \\
152 \\
54\end{array}$ & $\begin{array}{r}18,8 * * \\
16,3 * * \\
52 * \\
79 \text { * } \\
136 \% \\
191 * * \\
294: * \\
624\end{array}$ & $\begin{array}{c}* \\
\text { NS } \\
* \\
* \\
* \\
N S \\
N S\end{array}$ \\
\hline
\end{tabular}

Niveau de signification : $* * 1 \%, * 5 \%, N S=$ non significatif. 